\title{
Community-associated methicillin-resistant Staphylococcus aureus infections in a pediatric intensive care unit
}

\author{
Khaled Menif, Asma Bouziri, Ammar Khaldi, Asma Hamdi, Sarra Belhadj, Aida Borgi, Zahra Fitouri, Najla \\ Ben Jaballah
}

Pediatric intensive care unit, Children's Hospital of Tunis, Tunis, Tunisia

\begin{abstract}
Introduction: Community-associated methicillin-resistant Staphylococcus aureus (CA-MRSA) infection is an increasing problem worldwide. In developing countries, there is little data on CA-MRSA infection in children. This study reviewed the clinical features and outcomes of children admitted in a Tunisian pediatric intensive care unit with severe CA-MRSA infections.

Methodology: Retrospective chart review of patients coded for CA-MRSA over 10 years.

Results: There were $14(0.32 \%$ of all admissions $)$ patients identified with severe CA-MRSA infections. The median age was three months (range, 0.5-156 months). All patients had pulmonary involvement. Six children (42.8\%) developed septic shock. Two (14.3\%) patients had multifocal infection with deep venous thrombosis. Two (14.3\%) patients died.

Conclusions: Severe CA-MRSA pneumonia dominated presentation. The mortality of CA-MRSA infection in our series is lower than that previously reported.
\end{abstract}

Key words: methicillin-resistant Staphylococcus aureus; severe sepsis; pediatric intensive care unit; necrotizing pneumonia

J Infect Dev Ctries 2011; 5(8):587-591.

(Received 09 September 2010 - Accepted 25 November 2010)

Copyright (C) 2010 Menif et al. This is an open-access article distributed under the Creative Commons Attribution License, which permits unrestricted use, distribution, and reproduction in any medium, provided the original work is properly cited.

\section{Introduction}

Staphylococcus aureus is a frequent cause of infections in children, ranging from skin and soft tissue to invasive life-threatening infections [1]. Although community-associated methicillin-resistant $S$. aureus (CA-MRSA) isolates often are resistant only to methicillin and usually associated with skin and soft tissue infection, CA-MRSA isolates may also cause invasive and severe infections and even death in apparently healthy pediatric patients $[2,3]$. CA-MRSA infection in children is an increasing problem worldwide [4-8]. There is little published data on CA-MRSA infection in Tunisian children but reports from the United States suggest that MRSA accounts for up to $76 \%$ of all community-associated $S$. aureus isolates in some pediatric centers [5]. Children with severe CA-MRSA presenting to the pediatric intensive care unit (PICU) tend to develop multisystemic disease, either by direct invasion or toxin production, before the diagnosis is made and treatment initiated [9]. There is limited literature, with only single case reports or small patient groups, describing CA-MRSA in children admitted to an intensive care unit in the developed world [8, 10-11]. There have been no previous studies of CA-MRSA in children admitted in PICU in a developing country. This study evaluates the clinical features and mortality from CA-MRSA in those children who require intensive care management in a developing country.

\section{Methodology}

A retrospective review of clinical notes from all children with CA-MRSA admitted from 1 January 2000 to 31 December 2009 to a PICU was undertaken. The PICU is in a university affiliated children's hospital and provides intensive care services to a national pediatric population of 850,000 children younger than 15 years old. The hospital has 360 beds and the PICU has 14 beds. There were 4273 children admitted to the PICU during the study period. Neonates were only included if admitted to the PICU from the community. Children coded for MRSA were identified from the PICU database. All clinical notes were reviewed by one investigator using a standardized questionnaire that sought 
information on patient demographics, clinical findings, investigations, microbiology, and management in the PICU. Cases were included if blood or an isolate from a site that is normally sterile was positive for MRSA and/or the infection was community-associated. Community-acquired infection was defined by an isolate obtained within 48 hours of admission [5]. A severity of illness score (Pediatric Risk of Mortality Score PRISM) [12] was calculated for each patient. PRISM is a tool which uses 14 physiological variables measured at first contact with intensive care to assess severity of illness and give an index of risk of mortality for a population of children [12].

\section{Results}

Between 1 January 2000 and 31 December 2009, ninety-four patients with severe Staphylococcus aureus infection were admitted to the PICU of the Children's Hospital of Tunis; 14 (14.9\%) met the inclusion criteria. These 14 children accounted for $0.32 \%$ of the 4,273 admissions to the PICU over the study period. Table 1 shows their demographics and outcome data. More than fifty per cent $(56.4 \%)$ of the PICU admissions for CA-MRSA happened during 2008 and 2009, and $71.4 \%$ of hospitalizations occurred outside of the normal influenza season, which extends from November to March in Tunisia.

The median age of infection was three months (range, 0.5-156 months) with a predominance of infants under three months $(57 \%)$. Males accounted for five cases (35.7\%). The median PRISM was 12 , with a predicted mortality rate of $8.5 \%$. The observed mortality rate was $14.3 \%$ (2 of 14), compared with an overall PICU mortality rate during the study period of $16 \%$. The mean PICU stay of severe CA-MRSA cases was 14 days (range, one to 39 days), compared with our overall average PICU stay of 7.9 days. All children were transferred to the PICU following clinical deterioration on the ward after a mean delay of $2.9 \pm 2.3$ days (range: one to seven days). Reasons for ICU admission were respiratory failure requiring ventilation (71.4\%) and septic shock (28.6\%), although several children required multiple interventions.

All children had pulmonary involvement. Eleven

Table 1: Severe CA-MRSA Infections in PICU: Epidemiologic data

\begin{tabular}{|c|c|c|c|c|c|c|c|}
\hline $\begin{array}{l}\text { Case/month } \\
\text { and year of } \\
\text { admission }\end{array}$ & $\begin{array}{c}\text { Age } \\
\text { (months)/sex }\end{array}$ & PRISM & $\begin{array}{c}\text { Infected } \\
\text { site } \\
\text { culture } \\
(+)\end{array}$ & $\begin{array}{l}\text { Antibiotic } \\
\text { treatment }\end{array}$ & $\begin{array}{c}\text { Ventilation/Inotrope } \\
\text { (days) }\end{array}$ & $\begin{array}{l}\text { PICU } \\
\text { (days) }\end{array}$ & Outcome \\
\hline 1/09-2009 & 9/M & 8 & Lung & Teicoplanin & 2 & 4 & Survived \\
\hline 2/09-2009 & $157 / \mathrm{M}$ & 18 & Bld/joint & Vanc/Gent & $8 / 2$ & 9 & Died-refractory shock \\
\hline $3 / 12-2008$ & $7 / \mathrm{F}$ & & Lung & Vanc/Gent & $20 / 4$ & 29 & Survived \\
\hline 4/09-2008 & $5 / \mathrm{M}$ & & Bld & Fosf/Ctx/Gent & $1 / 1$ & 1 & Died-refractory shock \\
\hline $5 / 06-2006$ & $.5 / \mathrm{F}$ & & Lung/Bld & Fosf/Ctx/Gent & $9 / 5$ & 15 & Survived \\
\hline 6/11-2001 & $3.5 / \mathrm{F}$ & 18 & $\mathrm{Bld} / \mathrm{CSF}$ & Fosf/Ctx/Gent & 2 & 39 & Survived \\
\hline 7/09-2008 & $2 / \mathrm{F}$ & 13 & Lung & Teicoplanin & 10 & 11 & Survived \\
\hline $8 / 10-2006$ & $2 / \mathrm{F}$ & 33 & Lung & Fosf/Ctx/Gent & $5 / 2$ & 18 & Survived \\
\hline 9/09-2003 & $16 / \mathrm{F}$ & 5 & Lung & Fosf/Ctx/Gent & 12 & 26 & Survived \\
\hline $10 / 09-2008$ & $21 / F$ & 12 & Lung & Fosf/Ctx/Gent & 1 & 3 & Survived \\
\hline 11/10-2008 & $1 / \mathrm{F}$ & 5 & Lung & Teicoplanin & 5 & 7 & Survived \\
\hline $12 / 12-2007$ & $3 / \mathrm{M}$ & 9 & Lung/Bld & Vanc/Gent & 1 & 8 & Survived \\
\hline 13/12-2007 & $3 / \mathrm{M}$ & 15 & Lung & Vanc/Gent & 15 & 18 & Survived \\
\hline 14/04-2001 & $11 / \mathrm{F}$ & 3 & Lung & Fosf/Ctx/Gent & 16 & 9 & Survived \\
\hline
\end{tabular}

M, male; F, female; Bld, blood; Vanc, vancomycin; gent, gentamicin; fosf, fosfomycin; Ctx, cefotaxime; CA-MRSA, community-acquired methicillin resistant Staphylocoocus aureus PICU, pediatric intensive care unit; PRISM, Pediatric Risk of Mortality score. 
(78.6\%) had pneumonia on chest radiographs. Pleural drains were required in 13 of 14 children with empyema $(n=4)$, pyopneumothorax $(n=4)$, or pneumothorax $(n=5)$. Pleural drains were unilateral in nine patients and bilateral in four patients. All children required ventilation for a mean of 7.64 (SD 6.28 , range: $1-20$ ) days.

Six children (42.8\%) developed septic shock (defined as inadequate tissue perfusion from sepsis despite adequate filling) and five required inotropic with vasopressor support (dobutamine 10-20 $\mu \mathrm{g} / \mathrm{kg} / \mathrm{min}$, noradrenaline $0.5-4 \mu \mathrm{g} / \mathrm{kg} / \mathrm{min}$ ). The mean duration of inotrope and vasopressor support was 2.8 (SD 1.6, range: 0 - 5) days. The initial white blood cell count ranged from $1600 / \mathrm{mm} 3$ to 35 $000 / \mathrm{mm} 3$. Five patients were leukopenic on admission, one of whom died. All patients had elevated C-reactive protein (mean: $198.32 \pm 103.56$ $\mathrm{mg} / \mathrm{L}$, range: $55-484$ ), and the mean platelet count was 484428/mm3 (range: 16000-1124000 /mm3). Hyponatremia $(<130 \mathrm{mmol} / \mathrm{L})$ was a common feature encountered in nine $(64.3 \%)$ patients (mean: $123 \pm 6.52 \mathrm{mmol} / \mathrm{L}$, range: 112-128). Renal failure occurred in one patient, who had not required renal dialysis. A coagulopathy was seen in one child on presentation, and six progressed to multisystem organ failure. Of the 14 children, two $(14.3 \%)$ had multifocal infection.

One child aged 13 years had multiple joint involvement with three sites affected simultaneously (right knee, elbow, and left wrist) and required surgical drainage. The right knee septic arthritis was complicated by a femoral vein thrombosis with bilateral nodular densities consistent with septic emboli seen on chest radiographs.

Another child initially developed a preseptal cellulitis which was complicated by MRSA bacteremia, zygomatic bone osteomyelitis, cavernous septic thrombosis, meningitis and septic pulmonary localization. Blood culture was positive for MRSA in five children, with blood culture alone in two children. The content of thoracocentesis fluid grew MRSA in eleven patients; four grew MRSA from tracheal aspirates, and MRSA was isolated from either CSF or joint aspirate in two additional cases. The initial antibiotic started on admission to either the PICU or to hospital was inappropriate in 11 (78.6\%) children. The initial antibiotic was ceftriaxone in five children, flucloxacillin in four children, and amoxicillin-clavulanic acid in two children. The treatment regime for these children was changed, based on culture and susceptibility results to the combination of fosfomycin, cefotaxime and gentamicin in seven cases, vancomycin and gentamicin in four cases, and teicoplanin in three cases. Eight children received antibiotics for more than 15 days with a mean duration of $18.4 \pm 11.28$ days (range: 15-42 days). It was not possible to determine duration of antibiotics in six children who were transferred to other hospitals after PICU discharge. The MRSA isolates from these patients had a similar antibiotic susceptibility pattern: all were susceptible by disk diffusion to vancomycin, fosfomycin, gentamicin, and trimethoprimsulfamethoxazole. Only two MRSA isolates showed clindamycin resistance and four MRSA isolates were resistant to erythromycin.

\section{Discussion}

Our results, collected over 10 years from a single institution, differ significantly from prior literature reports of severe CA-MRSA infection in children. The main differences include: substantially lower mortality $(14.3 \%$ compared to the range of $30-50 \%$ previously published [11,13]), and a younger population (median age of three months versus 13-14 years in previous reports $[11,14])$, the predominance of the pulmonary involvement, and the rarity of musculoskeletal disease.

The most likely explanation of the differences from other published series is the case definition. We specifically used the criteria available in usual clinical practice, the antibiotic susceptibility pattern, to define our cases. Antibiotic susceptibility patterns also do not necessarily predict whether the isolate is a Panton-Valentine leukocidin (PVL)-producing strain [15]. PVL production varies significantly among different clinical isolates [16], suggesting other factors affect the phenotype and therefore the need for ICU care. While debate continues regarding whether the PVL toxin is the most important virulence factor $[17,18]$, presence of PVL appears to be an efficient marker for the more virulent strains [13]. The majority of our patients had lung necrosis and/or rapidly progressive pleural effusions. Since deterioration occurred when patients did not receive an antibiotic known to inhibit exotoxin production [19], antibiotic resistance patterns did clinically select patients with a high probability of toxin-producing CA-MRSA strains. However, the present study lacks molecular genetic analysis of the strains to support this hypothesis.

The lower mortality found in our study has three possible explanations. The first is again case 
definition. A previously published case series focused on cases accumulated by a center with specialized research interest in PVL-producing CA-MRSA [13]. The case series of Gillet et al. included 50 cases over nine years from 32 hospitals in nine countries, suggesting a significant selection bias [13]. A second possible explanation is the lack of seasonal variation (71.4\% occurred out of the flu season), suggesting that antecedent influenza was not a necessary feature of CA-MRSA pneumonia. In contrast, previous studies have suggested a significant relationship between lethal CA-MRSA pneumonia and preceding influenza [20,21]. Indeed, the interaction between influenza and CA-MRSA may either result in a highly lethal combination with unique clinical features or may stem from a more susceptible host genetic predisposition [22]. Unfortunately, this study lacks virological investigation to support either hypothesis. The last possible explanation for the mortality divergence is differences in treatment. Half of our patients (7/14) had received an empirical combination of parenteral fosfomycin, cefotaxime and gentamicin that achieved reasonable pulmonary, bone and brain penetration and was empirically active against $S$. aureus (including CA-MRSA strains). Fosfomycin, by inhibiting the production of penicillin binding protein $\mathrm{PBP} 2 \mathrm{a}$, which is involved in methicillin-resistance, restores the susceptibility of the MRSA strains to cefotaxime. This association allows rapid and total eradication even in the presence of a high bacterial inoculum. Finally, the rarity of musculoskeletal infections in this case series $(2 / 14)$ contributes to the better prognosis since more severe complications such as deep venous thrombosis are seen in patients with musculoskeletal infections caused by CA-S. aureus isolates, particularly when carrying the PVL genes [23]. Our patients with CAMRSA infection also did not have risk factors: none had concurrent skin lesions and all were immunocompetent.

In conclusion, despite necrotizing features, we found that the mortality due to CA-MRSA infection in our study populations was not as high as reported in other studies. Our treatment strategy may explain this better outcome. CA-MRSA is not necessarily a post-influenza infection. No clinical factors are highly predictive of CA-MRSA but suspicion should be raised by the radiographic features of necrotizing pneumonia and rapidly increasing pleural effusions. The increasing penetration of CA-MRSA in the community requires the dissemination of information to primary care providers about the potential severity of this infection, methods for rapid and accurate diagnosis, and the need to rapidly implement appropriate empiric and definitive treatment regimens.

\section{References}

1. Sattler CV and Correa AG (2004) Coagulase-positive staphylococcal infections (Staphylococcus aureus). In: Feigin RD, Cherry JD, Demmler GJ, Kaplan SL, editors. Textbook of Pediatric Infectious Diseases. Philadelphia: PA: Saunders. 1099-1129.

2. Martinez-Aguilar G, Hammerman WA, Mason EO Jr, Kaplan SL (2003) Clindamycin treatment of invasive infections caused by community acquired, methicillin-resistant and methicillin-susceptible Staphylococcus aureus in children. Pediatr Infect Dis J 22: 593-598.

3. Centers for Disease Control and Prevention (1999) Four pediatric deaths from community-acquired methicillinresistant Staphylococcus aureus-Minnesota and North Dakota, 1997-1999. JAMA 282: 1123-1125.

4. Ochoa T, Mohr J, Wanger A, Murphy R, Heresi G (2005) Community-associated methicillin-resistant Staphylococcus aureus in pediatric patients. Emerg Infect. Dis 11: 966-968.

5. Kaplan SL, Hulten KG, Gonzalez BE, Hammerman WA, Lamberth L, Versalovic J, Mason EO Jr (2005) Three-year surveillance of community-acquired Staphylococcus aureus infections in children. Clin Infect Dis 40: 1785-1791.

6. Gonzalez BE, Hulten KG, Dishop MK, Lamberth LB, Hammerman WA, Mason EO Jr, Kaplan SL (2005) Pulmonary manifestations in children with invasive community-acquired Staphylococcus aureus infection. Clin Infect Dis 41: 583-590.

7. Martinez-Aguilar G, Avalos-Mishaan A, Hulten K, Hammerman W, Mason E, Kaplan S (2004) Communityacquired, methicillin-resistant and methicillin-susceptible Staphylococcus aureus musculoskeletal infections in children. Pediatr Infect Dis J 23: 701-706.

8. Gonzalez BE, Martinez-Aguilar G, Hulten KG, Hammerman WA, Coss-Bu J, Avalos-Mishaan A, Mason EO Jr, Kaplan SL (2005) Severe staphylococcal sepsis in adolescents in the era of community-acquired methicillin-resistant Staphylococcus aureus. Pediatrics 115: 642-647.

9. Jensen AG (2002) Importance of focus identification in the treatment of Staphylococcus aureus bacteraemia. J Hosp Infect 52: 29-36.

10. Miles F, Voss L, Segedin E, Anderson BJ (2005) Review of Staphylococcus aureus infections requiring admission to a paediatric intensive care unit. Arch Dis Child 90: 12741278.

11. Castaldo ET, Yang EY (2007) Severe sepsis attributable to community-associated methicillin-resistant Staphylococcus aureus: An emerging fatal problem. Am Surg 73: 684-687.

12. Pollack MM, Ruutiman UE, Getson PR (1988) Pediatric risk of mortality (Prism) score. Crit Care Med 16: 1110-1116.

13. Gillet Y, Vanhems P, Lina G, Bes M, Vandenesch F, Floret D, Etienne J (2007) Factors predicting mortality in necrotizing community-acquired pneumonia caused by Staphylococcus aureus containing Panton-Valentine leukocidin. Clin Infect Dis 45: 315-321.

14. Creel AM, Durham SH, Benner KW, Alten JA, Winkler MK (2009) Severe invasive community-associated methicillin- 
resistant Staphylococcus aureus infections in previously healthy children. Pediatr Crit Care Med 10: 323-327.

15. Naimi TS, LeDell KH, Como-Sabetti K, Borchardt SM, Boxrud DJ, Etienne J, Johnson SK, Vandenesch F, Fridkin S, O'Boyle C, Danila RN, Lynfield R (2003) Comparison of community- and health care-associated methicillin-resistant Staphylococcus aureus infection. JAMA 290: 2976-2984.

16. Hamilton SM, Bryant AE, Carroll KC, Lockary V, Ma Y, McIndoo E, Miller LG, Perdreau-Remington F, Pullman J, Risi GF, Salmi DB, Stevens DL (2007) In vitro production of Panton-Valentine leukocidin among strains of methicillinresistant Staphylococcus aureus causing diverse infections. Clin Infect Dis 45: 1550-1558.

17. Labandeira-Rey M, Couzon F, Boisset S, Brown EL, Bes M, Benito Y, Barbu EM, Vazquez V, Höök M, Etienne J, Vandenesch F, Bowden MG (2007) Staphylococcus aureus Panton- Valentine leukocidin causes necrotizing pneumonia. Science 315: 1130-1133.

18. Bubeck Wardenburg J, Bae T, Otto M, Deleo FR, Schneewind O (2007) Poring over pores: alpha-hemolysin and Panton-Valentine leukocidin in Staphylococcus aureus pneumonia. Nat Med 13: 1405-1406

19. Stevens DL, Ma Y, Salmi DB, McIndoo E, Wallace RJ, Bryant AE (2007) Impact of antibiotics on expression of virulence-associated exotoxin genes in methicillin-sensitive and methicillin-resistant Staphylococcus aureus. J Infect Dis 195: 202-211.

20. Hageman JC, Uyeki TM, Francis JS, Jernigan DB, Wheeler JG, Bridges CB, Barenkamp SJ, Sievert DM, Srinivasan A, Doherty MC, McDougal LK, Killgore GE, Lopatin UA,
Coffman R, MacDonald JK, McAllister SK, Fosheim GE, Patel JB, McDonald LC (2006) Severe community-acquired pneumonia due to Staphylococcus aureus, 2003-04 influenza season. Emerg Infect Dis 12: 894-899.

21. Gillet Y, Issartel B, Vanhems P, Fournet JC, Lina G, Bes M, Vandenesch F, Piémont Y, Brousse N, Floret D, Etienne J (2002) Association between Staphylococcus aureus strains carrying gene for Panton-Valentine leukocidin and highly lethal necrotising pneumonia in young immunocompetent patients. Lancet 359: 753-759.

22. Wunderink RG (2008) Another piece of the communityacquired pneumonia puzzle. Chest 134: 1112-1114

23. Nourse C, Starr M, Munckhof W (2007) Communityacquired methicillin-resistant Staphylococcus aureus causes severe disseminated infection and deep venous thrombosis in children: Literature review and recommendations for management. J Paediatr Child Health 43: 656-661.

\section{Corresponding author}

Asma Bouziri

Service de Réanimation Pédiatrique

Hôpital d'enfants de Tunis

Baab Saadoun, 1007 Jabbari

Tunis, Tunisie

Phone number: 0021698652314

Fax number: 0021671561614

Email: asma.bouziri@yahoo.fr

Conflict of interests: No conflict of interests is declared. 